\section{Identificación del comportamiento de escolares y padres la familia respecto al mantenimiento de la salud bucal}

Identification of the behavior of students and parents regarding the family to maintain oral health.

\section{Resumen}

El objetivo del estudio fue determinar el comportamiento de escolares, padres de farnilia y docentes, en el mantenimiento de la salud bucal del niño. La investigaciòn Cuasiexperimental, cualicuantitativo se realizó en una muestra constituida por 46 niños y niñas de 6 años de edad, donde se evaluó condición de salud bucal inicial y final (caries inicial y manifiesta), se midió el indice de placa; también se determinó conocimientos y significados en salud bucal de los padres y/o madres de familia, igualmente de decentes. Se aplicó un modelo de capacitación para incrementar conocimientos y reconceptualización de saberes en salud bucal, con escenarios de aprendizaje participativos en docentes y padres, asimismo se realizo atención preventiva a las niñas y niños con énfasis en dentición permanente. Los resultados mostraron diferencias antes y después de la capacitación. La caries manifiesta mostró cambios significativos ( $p$-valor 0,0 ) despues de la capacitación y atenciòn preventiva de un $61 \%$, pasaron a ser caries manifiesta tratada en un $54 \%$, siendo un $7 \%$ la caries manifiesta no tratada. El porcentaje de niños con obturaciones al inicio de la intervención fue de un $4,3 \%$, despues de la capacitación y atenciòn preventiva en las caries manifiesta se elevó a un $58,6 \%$ mostro cambios significativos (p-valor) 0,00; el número de primeras molares protegidas con sellantes fue significativo ( $p$-valor 0,00 ), de un $0 \%$ inicial a un $95,6 \%$; el indice de higiene oral antes y despues de la capacitación hallo significancia ( $\mathrm{p}$-valor 0,00$)$; los niños sin placa bacteriana se incrementaron de un $0 \%$ al $6,5 \%$; los niños con deficiente higiene grado 3 , pasaron a tener una higiene regular conformando un $84,8 \%$. En lo referido a la capacitación en prácticas de salud bucal de los padres y madres de familia después de la intervención se halló diferencias estadísticamente significativa ( $p$-valor 0,00$)$. Se concluye que el acceso a la salud bucal de los niños y niñas en edades tempranas, depende de barreras culturales en colectivo tanto en la familia como en la escuela. El acceso a servicios básicos con un enfoque preventivo promocional participativo promueve un cambio de actitud del niño y niña y su entorno.

\section{Abstract}

The aim of this study was to determine the behavior of students, parents and teachers in maintaining the oral health of children. Quasi-experimental research, cualieuantitativo was conducted in a sample of 46 children aged 6 years old, which evaluated oral health status of the initial and final (initial and manifest caries) was measured by the plaque index also was determined knowledge and meanings in oral health of the parents or mothers, both teachers. Implemented a training model for enhancing knowledge and reconceptualization of knowledge in oral health, with scenarios in participatory learning teachers and parents also will perform preventive care to children with emphasis on permanent teeth. The results showed differences before and after training. Manifest caries showed significant changes ( $p$-value) 0.0 after training and preventive care of $61 \%$, became manifest caries treated by $54 \%$ and $7 \%$ untreated caries says. The percentage of children with fillings at the beginning of the intervention was $4.3 \%$, after training and preventive care in the manifest caries rose to $58.6 \%$ showed significant changes ( $p$-value) 0.0 , the number first molars with sealants was significantly protected ( $p$-value) 0.00 , an initial $0 \%$ to $95.6 \%$, the index of oral hygiene before and after training found significance (p-value) 0.0 ; children plaque increased from $0 \%$ to $6.5 \%$, children with poor hygiene grade 3 , they moved to take up a regular hygiene $84.8 \%$. In terms of training in oral health of parents and mothers after the intervention was found statistically significant differences (p-value) 0000 . We conclude that access to oral health of children at early ages, depends on collective cultural barriers in both the family and at school. Access to basic services with a preventive approach promotes a participatory promotional change of attitude of child and its environment.

\section{Artículo Original}

\section{Lita Ortiz Fernández, ${ }^{1}$ Margot Gutiérrez Have, ${ }^{1}$ Hilda Moromi Nakata, ${ }^{2}$ Katia Medina Calderón,' Jorge Villavicencio Gastelú. ${ }^{3}$}

Docentes del Departamento Académico de Estomatología Biosocial. UNMSM

2 Docente del Departamento Académico de Ciencias Básicas, UNMSM

Docente del Departamento Académico Médico Quirúgico. UNMSM

Correo electrónico: limaoff@yahoo.es

Correspondencia:

Lita Ortíz Fernández

Facultad de Odontología. UNMSM. Av. Germán Amézaga s/n. Lima 1. Perú

Teléfono: 999368035

Correo electrónico: limaoff@yahoo.es

Palabras Clave: Promoción y prevención en salud bucal, salud bucal escolares, salud bucal padres y docentes.

Key words: Promotion and prevention in oral health, school oral health, oral health educators and parents.

\section{Introducción}

Los primeros seis años de vida es el período de mayor vulnerabilidad física, $y$ a la vez de dependencia de otros para la atención de las necesidades básicas como abrigo, higiene, alimentación, etc. Ello se expresa en los mayores niveles de mortalidad, morbilidad y desnutrición en relación a otras etapas de vida, por lo que la vigilancia, el adecuado y oportuno tratamiento de estos aspectos son fundamentales. Se sabe que no sólo la desnutrición afecta el desarrollo; las enfermedades dismi- 
nuyyen el apetito impiden la absorción de los nutrientes, la repetición de estas enfermedades debilitadoras entre los niños de las comunidades pobres, constituyen las principales causas de retraso del desarrollo. En esta etapa de vida se forman hábitos saludables esenciales para la vida personal y en relación con otros, como los de alimentación adecuada, higiene y orden personal, convivencia social.

Los programas de atención a edades tempranas cumplen un rol preventivo al permitir mantener a los niños en salud o a una detección oportuna de dificultades de diferente tipo; la participación de la familia y la escuela es vital en la aplicación de los modelos de capacitación proponiendo cambios de comportamiento, desarrollando procesos cognitivos, con participación activa, fortaleciendo las relaciones con en el sistema de salud."

La salud bucal influye en la autoestima del niño y la niña, en la capacidad para alimentarse, en la nutrición y la comunicación, es decir interviene en los aspectos biológicos, psicológicos y sociales, por ende en el estado de salud general del niño.

Los Servicios de salud con enfoques preventivos, articulados con otros sectores como Educación, Cómunidad y la familia son necesarios para dar respuesta a los problemas de salud pública. Intervenir en la Escuela permite enfrentar los graves problemas de salud del niño y la niña: La evaluación y monitoreo de los problemas de salud de este grupo étario; las estrategias participativas y problematizadoras para la capacitación a su entorno para generar actitudes y respuestas positivas; la atención a las desigualdades, valoración a la accesibilidad de los servicios, conformación de redes en los distintos niveles de servicios tanto en atención primaria como especializada, permiten resolver y mantener la salud de los niños y niñas desde edades tempranas para mejorar su calidad de vida. ${ }^{1,2}$ Se apunta a un nuevo concepto de salud, no como un objetivo en sí mismo, sino como una característica de la vida cotidiana.

El presente estudio tiene como objetivo determinar el comportamiento de las variables sociales de la familia y la escuela en la participación indirecta para el mantenimiento integral de la salud bucal del niño y niña de seis años de edad, con un enfoque de promoción y prevención de salud.

\section{Material y Métodos}

La Investigación se realizó entre abril y noviembre de 2008, en el Distrito de San Martin de Porres y la Provincia Constitucional del Callao, en las instituciones educativas "José Andrés Rázuri" y "Rafael Belaúnde", respectivamente. Son Escuelas de educación básica, seleccionadas de modo que el material de estudio manifieste los siguientes criterios: escuelas urbanas, mixtas, con más de 200 escolares, estatales, con único turno de mañana, escolares de nivel socioeconómico bajo.

Las unidades de observación fueron los niños y niñas de seis años de edad. Las unidades de información fueron los padres/madres de familia y docentes de las instituciónes educativas. Se obtuvo el consentimiento informado y de participación de los padres y madres de familia, asimismo de los directivos y docentes de las dos instituciones educativas.

\section{Fases del estudio}

\section{Primera Fase:}

1. Diagnóstico inicial clínico sobre estado de salud bucal en los niños y niñas en cuanto a caries dental y presencia de placa bacteriana. Para la evaluación se uso luz natural, espejos bucales planos. Se entrenó a dos odontólogas para el manejo de los criterios caries inicial, ${ }^{3}$ caries manifiesta ${ }^{4}$ índice de placa. ${ }^{5} \mathrm{La}$ confiabilidad intraexaminador fue determinada mediante la aplicación del coeficiente de kappa obteniendose 0,91 para el índice de placa y el 0,89 para caries. La caries inicial se registró cuando se observó desmineralización de la superficie dental con pérdida de translucidez, ${ }^{3}$ y se considero caries manifiesta sin tratar según Koch, ${ }^{4}$ quien la define como el mínimo nivel que puede ser verificado en una cavidad o fisura detectable por sondeo o con retención del explorador bajo ligera presión. Se evaluó la higiene oral utilizando el índice de placa de Sillnes y Loe, ${ }^{5}$ se evaluaron los dientes deciduos 54-64-74.84-81-71; no se tomó en cuenta las primeras molares permanentes porque el $50 \%$ no llego a oclusión aún.

2. Validación del material de la encuesta. Se realizó una prueba piloto a 10 padres de familia (anónimos) de una escuela que no participó en el estudio, con caracteristicas equiparables a las de la población investigada. Se realizaron ajustes en cuanto al lenguaje y comprensión de cada una de las preguntas.

3. Aplicación inicial de una encuesta semiestructurada para identificar nivel de conocimientos (14 preguntas referidas a información en higiene bucal, medidas preventivas, frecuencia visita al odontólogo, tipos de denticiones, importancia primeras molares permanentes, loncheras saludables) y significados sobre salud bucal a las madres y padres de familia, a los docentes se les identificó nivel de conocimientos sobre salud bucal. La encuesta se cumplió en 25 minutos, en presencia de los investigadores, que solucionaron dudas.

\section{Segunda Fase:}

1. Aplicación de una propuesta educativa de capacitación, incorporando los resultados de la encuesta inicial.

2. Se elaboraron tres talleres de capacitación (vivenciales, con uso de material didáctico-lúdico) dos para padres - madres de familia y uno para docentes, teniendo en cuenta las debilidades y fortalezas de la encuesta inicial e información socioeconómica.

3. Se capacitó a los padres y madres de familia y docentes. aplicando estrategias basadas en el aprendizaje significativo, con metodologia participativa, se buscó la reconceptualización de un nuevo concepto de prevención en salud bucal, con escenarios de aprendizaje y participación activa buscando un aprendizaje penetrante no un simple aumento de conocimientos, enlazándolo con su vida misma, asumiendo un compromiso personal.

4. Se dio acceso a la atención clínica odontológica a los niños y niñas con autorización de sus madres o tutores, con un enfoque preventivo. Se aplicó sellantes en primeras molares permanentes y temporales, se obturaron caries manifiesta reversibles en dentición decidua, se aplicó barniz fluorado, se realizó control mecánico de placa bacteriana. 


\section{Tercera Fase}

1. Aplicación final de encuesta semiestructurada para identificar nivel deconocimientos y significados sobre salud bucal, después de la capacitación a padres, madres de familia y docentes.

2. Se realizó una segunda evaluación clínica odontológica después de la intervención preventiva utilizando los mismos criterios de la primera fase.

Las fases del estudio antes y después de la intervención entre la asociación de las variables clínicas antes y después de la atención se evaluó mediante el test de Wilcoxon, con un nivel de significancia del $5 \%$. Los significados de salud bucal de los padres y madres de familia y docentes, se evaluó cualitativamente, se diferenció las concepciones más explicativas de sus discursos un antes y un despuès.

\section{Resultados}

En el estudio se incluyeron a padres y madres de familia que asistieron a la capacitación en forma regular. El $82 \%$ era del sexo femenino y el $8 \%$ masculino, tenían un rango de edad de 22 a 54 años y un promedio de 36,9 años. El 61 $\%$ tenía grado de instrucción primaria, el $24 \%$ secundaria, el $8 \%$ instrucciòn superior no universitaria y el el $7 \%$ instrucción superior. En cuanto a la ocupación, el $78 \%$ era ama de casa, el $18 \%$ tenía trabajo eventual, el $7 \%$ trabajo fijo, el $4 \%$ no trabaja. En cuanto al acceso a la salud bucal de la familia, el $78 \%$ acudía al Centro de Salud del Ministerio de Salud, el $12 \%$ a los Policlinicos de la Seguridad Social, el $5 \%$ a los Hospitales Itinerantes, el $3 \%$ a los Centros de las Fuerzas Armadas, el $2 \%$ en forma privada.

Los padres y madres de familia de la institucion educativa Andrés Rázuri participó en un $90 \%$, mientras que las de Rafael Belaúnde participó un $60 \%$ en las tres fases, los cuatro docentes de la primera institución lo hicieron en un $90 \%$, los cuatro de la segunda institución participaron reducidamente, en $40 \%$, pese a las reiteradas convocatorias.

Del análisis de la información inicial suministrada por un $80 \%$ de los padres y madres de familia, destacan los significados de salud bucal de los padres y madres de familia relacionado la salud con la ausencia de enfermedad, tales como: 1. "Es cuando no tengo dolor de
dientes".
2. "Tengo buena salud bucal porque mastico bien, aunque hay veces me sangran mis encias".

3. "La picadura de los dientes y el sangrado de encías no son enfermedades que puedan afectar la salud general".

4. "Cuando tengo dolor de muelas tomo mi pastilla y ya está, puedo hacer mis cosas normalmente".

5. "No es necesario tener dientes sanos para tener buena salud".

6. "Los dientes de leche no tienen que cuidarse porque se mudan ${ }^{48}$.

7. "Las primeras molares permanentes salen a los 10 años".

8. "Yo llevo a mi hijo al dentista cuando tienen dolor de muelas y cuando se la saca ya está feliz".

8. "Tener una buena limpieza de los dientes es importante, yo me lavo los dientes cuando me acuerdo pero a mi hijita si le digo que lo haga después de que desayuna y almuerza".

10."Mi hijo ya va acumplir siete años, tiene bien sus dientes de leche, dos nomás tienen huequitos chiquitos, no le duele".

Posterior a la capacitación, se pudo identificar la integración del componente preventivo en los resignificados sobre salud bucal en el $78 \%$ de los padres y madres de familia, manifiestado a través de expresiones como:

1. "...es importante apoyar a mis hijos no sólo en la higiene dental sino también en el consumo de los alimentos que no producen caries..."

2. "...el cuidado y salud bucal de los hijos es en forma diaria y debe ser un hábito...p prometo hacerlo en casa..."

3. "...desconocía que la caries es una enfermedad contagiosa, tendre que curarme los dientes yo..."

4. "...las primeras muelas permanentes son importantes $y$ salen a los 6 años..."

5. "...he aprendido que los dientes de leche hay que cuidarlos hasta que salgan los fijos..."

6. "...tener salud en los dientes y en la boca intervienen para tener una buena salud de todo el cuerpo..."

7. "...tengo que llevar a mis hijos al dentista una vez al año... yo pensaba antes que debía llevarlos cuando les dolía algo... les calmaba el dolor de muelas dándole una pastilla que me daban en la farmacia..."

En el $80 \%$ de los docentes se identificó la integración del componente preventivo en los resignificados sobre salud bucal, manifestado en las expresiones:

1. "...los temas de causas de las enfermedades de la salud bucal fueron importantes.... trataremos de aplicar motivaciones con mas frecuencia en casa y en aula..."

2. "me capacité en como cuidar o prevenir la salud bucal...lo impulsaré en el aula..."

3. "...noscomprometemos a incentivar más la higiene bucal en los niños, recordándoles que se laven los dientes después de cada comida y de recreo..."

4. “...la situación de los niños en la escuela es dificil... desconociamos la realidad, pero con las informaciones que hemos obtenido y debatido nos damos cuenta que las causas de que los niños tengan caries van más allá. Todos estamos inmersos, no sólo es responsabilidad de la mamá, es de la familia, el centro de salud, los dentistas, la escuela...pondremos más esfuerzo".

Se encontró diferencias significativas en cuanto al grado de instrucción y nivel de conocimiento en salud bucal de los padres y madres de familia $\mathrm{p}$ valor 0,001 .

Asimismo mostró cambios significativos la condición libre de caries p-valor 0,001 , caries manifiesta p-valor 0,000 , el índice de obturaciones p-valor 0,00 antes y después de la intervenciòn (Fig 1). No se hallaron diferencias significativas en la caries inicial p-valor 0,739

Los escolares que participaron en el estudio fueron 46 , siendo $25(54 \%)$ de sexo femenino y 21 (46\%) masculino. En cuanto a la condición bucal de los niños y niñas examinados, $16(35 \%)$ estaba libre de caries dental antes y después de la capacitación de los padres y madres de familia. Los siete niños con caries inicial no presentaron cambios, más sí se observaron cambios en la caries manifiesta, siendo en total 28 niños con caries manifiesta, después de la intervención, pasaron a ser 25 (54\%) con caries manifiesta tratada, mostro cambios significativos ( $\mathrm{p}$-valor $0,0)$, siendo $3(7 \%)$ que tuvieron caries 
manifiesta no tratada; dos niños tenían obturaciones al inicio de la intervención (4\%) después de la intervención fueron 27 (58\%). Asimismo se identificó un $0 \%$ de primeras molares no protegidas con sellantes antes de la intervención, siendo luego su paso a un $95,6 \%$. Fig. 1 .

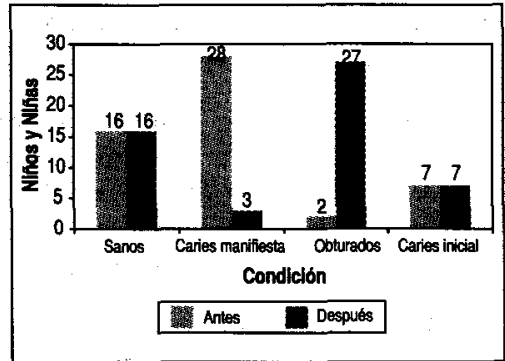

Fig. 1. Situación de la caries dental de los niños antes y después de la capacitación.

En cuanto a la presencia de placa bacteriana (Cuadro 1), se encontró hallazgos significativos ( $p$-valor), luego de la capacitación los niños y niñas sanos sin placa bacteriana se incrementaron de un $0 \%$ al $6,5 \%$; los niños con deficiente higiene tanto del grado 2 y 3 , pasaron a tener una higiene regular conformando un 84,8 $\%$, siendo el tránsito mayor de los de grado 3 de un $60,8 \%$ puesto que se mantuvieron como deficientes el 8,7 $\%$. La ingerencia de los padres capacitados se evidencio.

Cuadro 1. Presencia de placa bacteriana en niños y niñas de 6 años antes y después de la capacitación

\begin{tabular}{ccccc}
\hline \multirow{2}{*}{$\begin{array}{c}\text { Condi- } \\
\text { ción }\end{array}$} & \multicolumn{2}{c}{ Antes } & \multicolumn{2}{c}{ Después } \\
\cline { 2 - 5 } & $\mathrm{n}$ & $\%$ & $\mathrm{n}$ & $\%$ \\
\hline Sanos & 0 & 0 & 3 & 6,5 \\
Grado 1 & 2 & 4,3 & 3 & 6,5 \\
Grado 2 & 12 & 26,08 & 36 & 78,3 \\
Grado 3 & 32 & 69,56 & 4 & 8,7 \\
\hline Total & 46 & 100 & 46 & 100 \\
\hline
\end{tabular}

La relación de indice de higiene oral inicial y final es estadisticamente significativa con la prueba de contraste de Wilcoxson.

En lo concerniente a la capacitación en salud bucal de los padres y madres de familia (Fig. 2), antes de la intervención ninguno tenía un concepto y práctica buena, sólo 5 cambio a esa categoría, mientras que 40 pasaron a tener un concepto y práctica regular, de 9 que estuvieron en la etapa inicial. $\mathrm{El}$ calificativo de malo de 37 padres en la etapa inicial paso a 1 en la etapa final. Comparando el antes y despues de la capacitación, el nivel de los conceptos en salud bucal han mejorado de una manera estadísticamente significativa (p-valor) 0,000 .

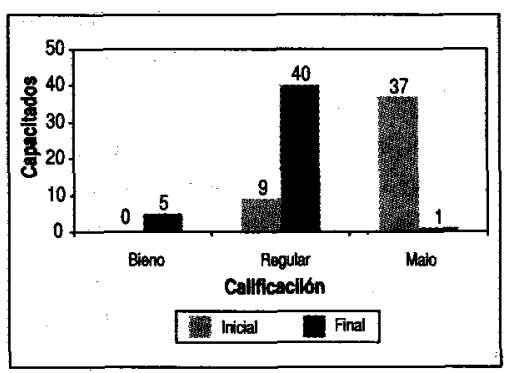

Fig. 2. Capacitación en prácticas en salud bucal de los padresde familia.

Se exploraron otras variables como grado de instrucción y edad de los padres y madres de familia, según condición bucal de sus hijos, las que no mostraron resultados estadísticamente significativos.

\section{Discusión}

Los resultados obtenidos al término de 8 meses de duración, indican que es posible lograr una disminución $y / o$ cambios en algunos indicadores de salud bucal de los niños y niñas de seis años de edad, luego de una capacitación e intervención temprana basada en la promoción y prevención, con la participación activa de la familia y la gestión proactiva en salud del equipo docente de las instituciones educativas. Se demostró que los padres y madres son los principales trasmisores de los hábitos de salud y de los comportamientos del niño y niña, coincidiendo con quienes realizaron estudios similares, involucrando a la familia, profesores y cuidadores en la adquisición de buenos hábitos higiénicos, los cuales son los factores primordiales para la creación de una cultura en salud bucal construida a partir de una metodología participativa problematizadora. ${ }^{6,7}$ La caries manifiesta mostró una alta prevalencia en los niños y niñas, asimismo se observó que ninguno tenia dientes obturados antes de aplicar la intervención, coincidendo con lo reportado por otro autores. ${ }^{6-10}$

Después de la atención clínica y la capacitación se produjo una reducción importante de niños con caries sin tratar y un aumento significativo de dientes obturados. Al relacionar la caries manifiesta antes y después, ésta fue estadisticamente significativa ( $p$-valor 0,0$)$ coincidiendo con otro estudio. ${ }^{9}$ La caries inicial se mantuvo igual antes y despúes, debido posiblemente a la intervención con medidas preventivas. Esto nos permite afirmar que la prevención primaria, en programas en escenarios saludables deben incorporar a los padres y madres de familia, con ello se contribuye a detener lesiones establecidas, y a mantener en salud a las primeras molares permanentes recien erupcionadas, obteniendo mejoras sustanciales en la salud bucal.

Al inicio del estudio se encontró que el $100 \%$ de los niñas y niños tenian placa bacteriana, coincidiendo con reporte donde el $90 \%$ tenian placa bacteriana. ${ }^{10} \mathrm{Al}$ comparar si hay asociación entre el antes y después de presencia de placa bacteriana, se identificó que fue estadísticamente significativo, el p-valor 0,0 los datos evidencian que el indice de placa bacteriana final tiene un nivel inferior al obtenido al inicio concordando con otro reporte. ${ }^{9}$ Algunos autores señalan que una política de cuidados de salud bucal orientados a la prevención podría ser mas ventajosa que los de enfoque curativos, y que son necesarios programas sistemáticos cuya base sea la educación, promoción de la salud en la familia y la escuela, unido al costo beneficio de aplicar medidas preventivas en niños sanos y sanos con riesgo a enfermar. ${ }^{1,11-14}$ Coincidiendo la capacitación con este enfoque, interviniendo a 16 escolares (35\%) sanos y sanos con riesgo a enfermar, donde el costo beneficio es diferente al intervenir a 30 niños (65 $\%)$ que están enfermos y enfermos con secuela.

Los conceptos y prácticas en salud bucal antes y después del programa ha mejorado de una manera estadísticamente significativa p-valor 0.000 en concordancia con otro estudio. ${ }^{9}$ Asimismo posterior a la implementación de las estrategias y acciones, se pudo identificar la integración del componente preventivo en los conceptos resignificados sobre salud bucal en padres y madres de familia y el desarrollo de sus capacidades de análisis, manifiesto a través de sus nuevas expresiones.

Después de la identificación de la condición del componente bucal de la salud de las niñas y niños; de los conocimientos y significados de salud bucal de los padres-madres defamilia y docentes, se implementaron estrategias mediadoras que posibilitaron despertar el interés en la responsabilidad y el compromiso de inculcar hábitos bucales saludables en los 
niños y niñas, padres $y / o$ madres de familia, mediante la participación en actividades de fomento y prevención desarrolladas en la institución educativa y reforzadas en el hogar. Coincidimos en la necesidad de desarrollar programas de salud bucal con la participación activa de los padres, ${ }^{72-13}$. Las estrategias mediadoras se sustento en lo lúdico y el constructivismo, donde el juego es la herramienta para expresar la adquisición de nociones y conceptos. ${ }^{212-13}$

La vinculación de los padres de familia y la comunidad a las actividades educativas de la escuela, es decisiva para elevar los aprendizajes de las niñas y niños, asimismo para fortalecer su calidad de vida. La cual depende de la gestión escolar, tanto pedagógica como institucional. Las Instituciones Educativas de zonas marginales tienen poca participación porque sobrellevan un deterioro constante, subsisten y como tal no están en condiciones de velar por la salud de sus educandos ni garantizar que estos adopten comportamiento y estilos de vida sanos, asimismo tienen que enfrentar un sistema de salud pasivo ajeno a esta realidad coincidiendo con otro estudio. ${ }^{13,14}$

\section{Conclusiones}

Se concluye que:

El estado de salud bucal de los niños y niñas mejoró significativamente en un $95 \%$ luego de la capacitación a los padres y madres de familia y docentes con estrategias participativas para la enseñanza aprendizaje, en la resignificación de los conceptos y en el mejoramiento de la higiene bucal de los niños.
Asimismo hubo mejoras con las acciones preventivas en el mantenimiento de la salud bucal de los niños sanos y los sanos con riesgo a enfermar y la recuperación de la salud bucal de los niños enfermos con caries dental:

La accesibilidad del niño y niña a una atención integral del componente bucal de la salud, integrando a su entorno familiar y escolar promueve un cambio de comportamiento.

\section{Agradecimiento}

Al Consejo superior de investigación de la UNMSM, por el financiamiento del presente estudio. Código 080502021.

\section{Referencias bibliográticas}

1. Gós L. Promoción de la salud, educación para la salud y comunicación social en salud: Especificidades, interfaces, intersecciones. Promotion \& Education: International Journal of Health Promotion and Education. 2000;7(4):8-12.

2. Conferencia en Alma-Ata sobre atención primaria de salud. OMS: Alma-Ata. 1978.

3. Grindefjord M. Caries development in children from 2.5 to 3.5 years of age. A longitudinal study. Caries Res 1993;27:505-510.

4. Koch G. Effect of sodium fluoride in dentrifice and mouthwash on incidence of dental caries in schoolchildren. Odontol Rev 1967;18:12-16.

5. Silness ], Löe H. Periodontal disease in pregnancy. Acla Odontol Scand 1964;22:121-128.

6. Löe H, Silness J. Periodontal disease in pregnancy. Acta Odontol Scand $1963 ; 21: 533-539$.
7. Navas R, AlvarezC, Rojas T.Metodología estratégica para la participación de los padres en los cuidados de la salud bucal de niños preescolares. $O D$, ene. 2005;2(1):22-33.

8. Wyne AH. Caries Prevalence, Severity, and Pattern in Preschool Children. I Contemp Dent Pract 2008; 9(3):24-31.

9. Rasquin $C$; Domínguez $M$; Alarcón M; Prieto I; Bellorín C. Cambios en la Valoración de la salud oral en los pacientes que acuden al Servicio odontológico del municipio Arismendi. Río Caribe. Estado Sucre 1998-2002. Acta Odontológica Venezolana: 2005;43(2): 9-12.

10. Segovia V. Dental caries experience and factors among preschoolers in southeastern Mexico: a brief communication. J Public Health Dent. 2006;66:88-91.

11. Ling Zhu, Poul Erik Petersen, HongYing Wang, Yin-You Biand, Oral health Knowledge, attitudes and behavior of children and adolescents in China, International Dental Journal. 2003;53(5):289-98.

12. Ortiz RA. Condiciones de vida y de Salud Bucal del escolarizado y su familia, Municipio Caroni, Estado Bolivar. 1992. Acta Odontológica Venezolana. 2000;38(1):18-36.

13. World Health Organization. Oral Health Surveys: Basic Methods. 3ru ed. Geneva, WHO. 1997.

California Strategic Plan for Parental Involvement in Education. California Department of Education 1992; and Building School-Family Partnerships for Learning. By Oliver C. Moles and Diane D'Angelo, US Department of Education, 1993.

Fecha de recepción: 15 abril 2009 Fecha de aceptación: 10 julio 2009 